\title{
Turbulent spectra and spectral kinks in the transition range from MHD to kinetic Alfvén turbulence
}

\author{
Y. Voitenko and J. De Keyser \\ Belgian Institute for Space Aeronomy, Ringlaan-3-Avenue Circulaire, 1180 Brussels, Belgium \\ Received: 5 April 2011 - Revised: 24 August 2011 - Accepted: 2 September 2011 - Published: 13 September 2011
}

\begin{abstract}
A weakly dispersive range (WDR) of kinetic Alfvén turbulence is identified and investigated for the first time in the context of the MHD/kinetic turbulence transition. We find perpendicular wavenumber spectra $\propto k_{\perp}^{-3}$ and $\propto k_{\perp}^{-4}$ formed in WDR by strong and weak turbulence of kinetic Alfvén waves (KAWs), respectively. These steep WDR spectra connect shallower spectra in the MHD and strongly dispersive KAW ranges, which results in a specific doublekink (2-k) pattern often seen in observed turbulent spectra. The first kink occurs where MHD turbulence transforms into weakly dispersive KAW turbulence; the second one is between weakly and strongly dispersive KAW ranges. Our analysis suggests that partial turbulence dissipation due to amplitude-dependent non-adiabatic ion heating may occur in the vicinity of the first spectral kink. The threshold-like nature of this process results in a conditional selective dissipation that affects only the largest over-threshold amplitudes and that decreases the intermittency in the range below the first spectral kink. Several recent counter-intuitive observational findings can be explained by the coupling between such a selective dissipation and the nonlinear interaction among weakly dispersive KAWs.
\end{abstract}

\section{Introduction}

Kinetic Alfvén waves (KAWs) are an extension of MHD Alfvén waves in the range of high perpendicular wavenumbers $k_{\perp}$ in the plane $\perp B_{0}$, where linear and nonlinear effects due to finite values of $k_{\perp} \rho_{\mathrm{p}}$ become significant ( $B_{0} \| z$ is the background magnetic field, $\rho_{\mathrm{p}}=V_{T p} / \Omega_{\mathrm{p}}$ is the proton gyroradius as we consider a hydrogen plasma) (Hasegawa and

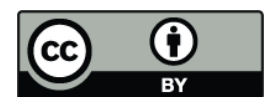

Correspondence to: Y. Voitenko (yuriy.voitenko@aeronomy.be)
Chen, 1976; Voitenko, 1998a). There are numerous observational and theoretical indications that MHD Alfvén turbulence in the solar wind cascades towards high $k_{\perp}$ and eventually reaches the KAW wavenumber range at the proton gyroradius scales, $k_{\perp} \rho_{\mathrm{p}} \sim 1$ (Leamon et al., 1999; Bale et al., 2005; Alexandrova et al., 2008a; Sahraoui et al., 2009, 2010). It is not yet certain what happens next with these KAWs: do they dissipate by heating the plasma (Leamon et al., 1999), or do they interact nonlinearly among themselves and cascade further towards higher $k_{\perp}$, ultimately reaching electron scales (Sahraoui et al., 2009, 2010; Alexandrova et al., 2009). Spectra up to electron scales with Cluster were first studied in the Earth's magnetosheath (Mangeney et al., 2006; Alexandrova et al., 2008b).

If the dissipation at ion scales is strong, the cascade should terminate in the vicinity of the spectral break $k_{\perp \mathrm{b}} \sim \rho_{\mathrm{p}}^{-1}$ and cannot reach electron scales, as was argued by Leamon et al. (1999), Howes et al. (2008), and Podesta (2009) using Landau damping estimates for KAWs. However, observations of power law spectra at $k_{\perp} \rho_{\mathrm{p}} \gg 1$ (Sahraoui et al., 2009, 2010; Alexandrova et al., 2009; Kiyani et al., 2009) suggest a continuation of the cascade that is consistent with the theoretical picture of KAWs (Bale et al., 2005; Schekochihin et al., 2009; Sahraoui et al., 2010). It has been envisaged that the nonlinear evolution and related wavenumber spectra in the range below $k_{\perp \mathrm{b}}$ are dominated by MHD-type nonlinear interactions among Alfvén waves, and that the spectra for $k_{\perp}>k_{\perp \mathrm{b}}$ are determined by KAW properties (Schekochihin et al., 2009).

One should note that the identification of the observed small-scale turbulence as KAWs is not unique. In particular, observations based on the k-filtering technique are controversial: Sahraoui et al. (2010) have shown that the properties of observed fluctuations are compatible with the KAW dispersion relation, whereas Narita et al. (2011) did not find KAWs. Relaxing the assumption of plane waves, Chen et al. (2010)

Published by Copernicus Publications on behalf of the European Geosciences Union and the American Geophysical Union. 
found that the turbulence between the ion and electron scales is not a pure KAW turbulence. Based on simulations, (Saito et al., 2010) concluded that not only KAW turbulence but also whistler turbulence may explain the very steep spectra observed recently at electron scales.

From an observational point of view, the nature of the turbulence changes at the spectral breaks $f_{\mathrm{b}}$ that separate shallower MHD spectra $\propto f^{-5 / 3}$ for $f<f_{\mathrm{b}}$ from steeper kinetic spectra with power indexes ranging from -2 to -4 for $f>f_{\mathrm{b}}$. Such breaks are observed in the solar wind by satellites ( $f$ is the frequency in the satellite frame). Because the solar wind velocity $V_{\mathrm{SW}}$ is much larger than the Alfvén velocity $V_{\mathrm{A}}$, the Alfvénic time variation $\omega_{\mathrm{A}} \sim k_{z} V_{\mathrm{A}}\left(k_{z}\right.$ is the wavenumber parallel to $B_{0}$ ) is usually much slower than the Doppler frequencies in the satellite frame $\omega_{\mathrm{d}}=k \cdot V_{\mathrm{SW}}$. Then the satellite-frame frequency spectra are dominated by the Doppler frequency, $2 \pi f=\left|k_{z} V_{\mathrm{A}}-\mathbf{k} \cdot \mathbf{V}_{\mathrm{SW}}\right| \sim\left|\mathbf{k} \cdot \mathbf{V}_{\mathrm{SW}}\right|$, representing wave-number spectra (Taylor hypothesis). As has been first shown by Matthaeus et al. (1990), followed by many others (see e.g. recent papers by Osman and Horbury (2009), Sahraoui et al. (2010), Luo and Wu (2010), and references therein), solar wind turbulence is dominated by large perpendicular wavenumbers $k_{\perp} \gg k_{z}$. In these conditions, satellites measure perpendicular wavenumber spectra, $f \propto k_{\perp}$, except for rare cases of $B_{0} \| V_{\mathrm{SW}}$, where frequency measures parallel wavenumber, $f \propto k_{z}$. The spectral break $f_{\mathrm{b}}$ is often associated with one of the proton kinetic scales, the proton gyroradius $\rho_{\mathrm{p}}$ or the proton inertial length $\delta_{\mathrm{p}}=V_{\mathrm{A}} / \Omega_{\mathrm{p}}$, such that the observed frequency of the break is $2 \pi f_{\mathrm{b}} \simeq V_{\mathrm{SW}} / \rho_{\mathrm{p}}$ or $V_{\mathrm{SW}} / \delta_{\mathrm{p}}$ (Leamon et al., 1999; Bale et al., 2005; Alexandrova et al., 2010; Sahraoui et al., 2010). Perri et al. (2010) show, however, that the break position is not sensitive to the radial dependence of the ion scales, whereas Markovskii et al. (2008) argue that the break position depends upon a combination of the scale and the turbulent amplitudes at that scale.

Because of the complex interplay between linear and nonlinear KAW dynamics, the theoretical interpretation of turbulence in the KAW range, its dissipation, and the related spectra, is still incomplete. In particular, a recent theoretical analysis by Podesta et al. (2010) argues that the KAW cascade is subject to collisionless Landau damping and cannot reach electron scales in solar wind conditions. However, using Cluster data, Sahraoui et al. $(2009,2010)$ and Alexandrova et al. $(2009,2010)$ have shown that the spectra extend to electron scales, with spectral slopes -1.7 and -2.8 in the MHD range and in the range between ion and electron scales, respectively. Between these $k_{\perp}^{-1.7}$ and $k_{\perp}^{-2.8}$ spectra, Sahraoui et al. (2010) also noticed much steeper $\propto k_{\perp}^{-4}$ spectra due to weakly/mildly dispersive KAWs with $0.6<k_{\perp} \rho_{\mathrm{p}}<2$ (see their Figs. 2, 3, and 6), and suggested that the KAW turbulence extends further down to electron scales. The same spectral form in the MHD/kinetic transition range, containing two spectral kinks with steeper spectra in between, can be seen in other recent studies (see e.g.
Chen et al. (2010), Fig. 1, and Smith et al. (2006), Fig. 1). Alexandrova et al. $(2009,2010)$ have demonstrated the universal character of the MHD $k_{\perp}^{-1.7}$ and kinetic $k_{\perp}^{-2.8}$ spectra and analyzed the non-universal transition between them. Kinetic spectrum ends up with a curved spectrum at electron scales, indicating dissipation.

Steep variable spectra in the same wavenumber range were observed before (Leamon et al., 1999), but without connection to shallower higher-wavenumber (higher-frequency in the satellite frame) spectra as these were unavailable. Such steep spectra were called "dissipation range" spectra and were associated with dissipation, mainly via kinetic ioncyclotron and Landau damping. However, the nature of the "dissipation range" and its spectra is not so clear. For example, recent observations of reduced magnetic helicity imply the presence of counter-streaming KAWs surviving the "dissipation range" rather than ion-cyclotron damping in it (Carbone et al., 2010).

Analyzing ACE spacecraft data, Smith et al. (2006) have found that larger spectral fluxes (as measured at $0.01 \mathrm{~Hz}$ ) are followed by steeper spectra in the "dissipation range" above the spectral break $f_{\mathrm{b}} \sim 0.3 \mathrm{~Hz}$. This counterintuitive observational fact is difficult to explain by ion-cyclotron and Landau damping. Smith et al. (2006) did not find any regular dependence of $f_{\mathrm{b}}$ on the cascade rate. Markovskii et al. (2008) studied the statistics and scaling of spectral breaks and concluded that their positions are determined by a combination of their scales and the turbulent amplitudes at that scales, which suggests a non-linear dissipation mechanism for the solar wind turbulence. Again, kinetic ion-cyclotron and Landau damping mechanisms would not lead to such behavior in the dissipation range.

Motivated by these findings, the present paper analyzes the wavenumber range that corresponds to the transition from MHD to KAW turbulence, with the focus on the properties of nonlinear KAW. We demonstrate that the observed spectral forms and steep spectra in the "dissipation range" can be explained by the nonlinear interaction of weakly dispersive KAWs without involving kinetic ion-cyclotron and Landau dissipation mechanisms. We will distinguish weak and strong turbulence by comparing linear and nonlinear time scales (or associated wave frequency $\omega_{k}$ and nonlinear interaction rate $\left.\gamma_{k}^{\mathrm{NL}}\right)$ at the given scale $k^{-1}$. If the linear time scale $\tau_{k}^{\mathrm{L}}\left(\tau_{k}^{\mathrm{L}} \sim 1 / \omega_{k}\right)$ is shorter than the nonlinear one $\tau_{k}^{\mathrm{NL}}$ $\left(\tau_{k}^{\mathrm{NL}} \sim 1 / \gamma_{k}^{\mathrm{NL}}\right)$, the perturbations have enough time to set up linear dispersion and polarization relations. In this case the energy exchange among perturbations is relatively slow, and the turbulence is weak. Since $\gamma_{k}^{\mathrm{NL}}$ increases with increasing amplitudes and $\omega_{k}$ increases with increasing $k_{z}$, the weak turbulence regime can be realized for sufficiently small amplitudes and sufficiently short parallel wavelengths. For larger amplitudes and/or longer wavelengths $\tau_{k}^{\mathrm{NL}}$ and $\tau_{k}^{\mathrm{L}}$ become comparable, and the so-called strong turbulence in the critical balance regime is realized: $\tau_{k}^{\mathrm{L}} \simeq \tau_{k}^{\mathrm{NL}}$ (Goldreich and 
Sridhar, 1995). Observations of intermittency in the inertial MHD range (Sorriso-Valvo et al., 1999; Salem et al., 2009) and in the range above the ion break (Alexandrova et al., 2008a) suggest the presence of a strongly turbulent fraction of fluctuations, but do not exclude the simultaneous presence of a weakly turbulent fraction, in particular in the MHD/kinetic transition range.

\section{Weakly dispersive $k_{\perp} \rho_{\mathrm{p}}<1$ range of KAW turbulence}

The rate of nonlinear interaction among highly oblique $k_{z} \ll$ $k_{\perp}$ KAWs has been calculated by maximizing the matrix element of the 3-wave KAW interaction (Voitenko, 1998a,b). In WDR, $k_{\perp}^{2} \rho_{\mathrm{p}}^{2} \ll 1$, for co-propagating KAWs

$\gamma_{k \uparrow \uparrow}^{\mathrm{NL}} \simeq 0.4 \Omega_{\mathrm{p}} \frac{V_{\mathrm{A}}}{V_{T p}}\left(k_{\perp} \rho_{\mathrm{p}}\right)^{3} \frac{B_{k}}{B_{0}}$,

where $B_{k}$ is the KAW amplitude at the (anisotropic) length scales $\lambda_{z}=2 \pi / k_{z}$ and $\lambda_{\perp}=2 \pi / k_{\perp}$, where $k_{z}$ and $k_{\perp}$ are parallel and perpendicular KAW wavenumbers. We account for the plasma compressibility, but neglected magnetic compressibility. Also, for simplicity we put $T_{e \|} / T_{p \perp}=1$. This temperature ratio reflects the relative importance of the parallel electron pressure and the perpendicular ion pressure/gyroradius effects in KAWs. With increasing $T_{e \|} / T_{p \perp}$ one would obtain higher interaction rates.

The nonlinear interaction rate for counter-propagating $k_{\perp} \rho_{\mathrm{p}} \ll 1 \mathrm{KAWs}$ is (Voitenko, 1998a,b)

$\gamma_{k \uparrow \downarrow}^{\mathrm{NL}} \simeq 0.3 \Omega_{\mathrm{p}} \frac{V_{\mathrm{A}}}{V_{T p}}\left(k_{\perp} \rho_{\mathrm{p}}\right)^{2} \frac{B_{k}}{B_{0}}$,

which is larger than $\gamma_{k \uparrow \uparrow}^{\mathrm{NL}}$ in WDR.

There is no explicit $k_{z}$-dependence of the KAW interaction rates Eqs. (1-2). The nonlinear KAW dynamics is thus driven by the energy exchange among short cross-field length scales, whereas the parallel scales follow the perpendicular ones kinematically in the weak turbulence regime (Voitenko, 1998a,b) or via critical balance in the strong turbulence regime (Schekochihin et al., 2009). The perturbation amplitude $B_{k}$ can be related to the omnidirectional spectral energy density $W_{k \perp}$ by $B_{k}=\sqrt{k_{\perp} W_{k \perp}}\left(W_{k \perp}\right.$ is defined such that $\int_{0}^{\infty} d k_{\perp} W_{k \perp}=$ total fluctuation energy per unit volume).

\subsection{Weak KAW turbulence}

The nonlinear interaction among co-propagating KAWs can be considered weak if their nonlinear rate Eq. (1) is less than the dispersive part of frequency: $\gamma_{k \uparrow \uparrow}^{\mathrm{NL}}<k_{z} V_{\mathrm{A}}\left(k_{\perp} \rho_{\mathrm{p}}\right)^{2}$ in isothermal plasmas. In this case, the conservation law for the generalized enstrophy (dispersive part of energy) applies, and the nonlinear interaction among co-propagating KAWs Eq. (1) establishes the perpendicular wavenumber spectra (Voitenko, 1998b):

$W_{k} \propto k_{\perp}^{-5} \quad$ (direct enstrophy cascade);
$W_{k} \propto k_{\perp}^{-4} \quad$ (inverse energy cascade).

For axially symmetric turbulence in the cross-field plane we can define a reduced omnidirectional spectral power $W_{k \perp}$ $=2 \pi k_{\perp} \int d k_{z} B_{k}^{2}$, such that $W=\int d k_{\perp} W_{k \perp}$. The energy exchange among different $k_{\perp}$ does not depend on $k_{z}$ (Voitenko, 1998a, Eqs. 6.1 and 6.2). Hence the reduced omnidirectional weakly turbulent 3-D power spectra Eqs. (3-4) behave as

$W_{k \perp} \propto k_{\perp}^{-4} \quad$ (direct enstrophy cascade),

$W_{k \perp} \propto k_{\perp}^{-3} \quad$ (inverse energy cascade)

The omnidirectional wavenumber spectra are those measured in the solar wind by satellites as 1-D Doppler frequency spectra if the solar wind velocity $V_{\mathrm{SW}} \nVdash B_{0}$. When the turbulence spectrum is not axisymmetric around $B_{0}$ (see e.g. Sahraoui et al., 2010, and references therein), the measured 1-D spectrum may have a larger power index, approaching $W_{k \perp} \propto k_{\perp}^{-5}$ in the extreme case of 1-D turbulence in the cross-field plane, $\propto k_{x}^{-5}$, if $x \nmid V_{\mathrm{SW}}$. This follows from polar angle averaging in the cross-field wavenumber plane. Accounting for this possibility, the steepest spectra produced by weakly dispersive KAW turbulence are

$W_{k \perp} \propto k_{\perp}^{-4} \div k_{\perp}^{-5}$

Nonlinear interaction among counter-propagating KAWs in the weakly turbulent regime, at a rate given by Eq. (2), produces omnidirectional spectra

$W_{k \perp} \propto k_{\perp}^{-2} \quad$ (direct enstrophy cascade),

$W_{k \perp} \propto k_{\perp}^{-1} \quad$ (inverse energy cascade)

that follow from 3-D spectra given by Voitenko (1998b). Therefore, counter-propagating KAW interactions cannot produce the steep spectra observed in the transition range.

At first sight, since $\gamma_{k \uparrow \downarrow}^{\mathrm{NL}} \gg \gamma_{k \uparrow \uparrow}^{\mathrm{NL}}$ in WDR, nonlinear interaction among counter-propagating KAWs appears to be a more efficient means of spectral transport than that due to co-propagating KAWs. However, the short (linear) correlation times among counter-propagating KAWs, $\tau_{\mathrm{c} \uparrow \downarrow}^{\mathrm{L}} \sim$ $\lambda_{z} / V_{\mathrm{A}}$, can reduce their interaction strength as compared to the co-propagating KAWs that remain in phase for a longer time: $\tau_{\mathrm{c} \uparrow \uparrow}^{\mathrm{L}} \sim\left(k_{\perp} \rho_{\mathrm{p}}\right)^{-2} \lambda_{z} / V_{\mathrm{A}} \gg \tau_{\mathrm{c} \uparrow \downarrow}^{\mathrm{L}}$ for $k_{\perp} \rho_{\mathrm{p}} \ll 1$. In such cases the nonlinear interaction among co-propagating KAWs will be dominant and the steepest omnidirectional spectra Eq. (7) are formed, as described above. When both co- and counter-propagating interactions are efficient, the resulting spectrum lies between the counter-propagating $\left(\propto k_{\perp}^{-2}\right)$ and co-propagating $\left(\propto k_{\perp}^{-4}\right)$ spectra. The relative importance of co- and counter-propagating KAW interactions, and the resulting spectral slope, will depend on what fraction of the MHD turbulent cascade will arrive at the MHD/KAW break with parallel wavelengths satisfying the condition of linear 
decorrelation, $\tau_{\mathrm{c} \uparrow \downarrow}^{\mathrm{L}} \sim \lambda_{z} / V_{\mathrm{A}}<1 / \gamma_{k \uparrow \downarrow}^{\mathrm{NL}}$. The larger this fraction, the steeper will be the resulting KAW spectrum.

The $k_{z}$ spectra of weak KAW turbulence are determined by the kinematics of three-wave resonant interactions (Voitenko, 1998b):

$W_{k \|} \propto k_{z}^{-1 / 2}$.

However, some non-kinematic factors, like finite resonance width, can make the parallel spectrum significantly different from Eq. (10). This point needs further investigation.

\subsection{Strong KAW turbulence}

In the strongly turbulent regime the nonlinear evolution time, $\tau_{k}^{\mathrm{NL}} \sim 1 / \gamma_{k}^{\mathrm{NL}}$, becomes equal to or shorter than the linear one, $\tau_{k}^{\mathrm{L}} \sim 1 /\left(k_{z} V_{\mathrm{A}} k_{\perp}^{2} \rho_{\mathrm{p}}^{2}\right)$. The enstrophy (dispersive part of energy) is not conserved any more because the nonlinear interaction does its job before the dispersive time mismatch comes into play. The $k_{\perp}$-spectrum can be found from the condition that the energy flux is constant (independent of $\left.k_{\perp}\right)$ :

$\varepsilon \sim B_{k}^{2} / \tau_{k}^{\mathrm{NL}}=$ const.

The nonlinear evolution time for co-propagating KAWs can be estimated as $\tau_{k \uparrow \uparrow}^{\mathrm{NL}} \simeq 1 / \gamma_{k \uparrow \uparrow}^{N L}$, where $\gamma_{k \uparrow \uparrow}^{\mathrm{NL}}$ is given by Eq. (1). From Eq. (11) we find the scaling for the fluctuating magnetic amplitude $B_{k} \propto k_{\perp}^{-1}$, which results in the omnidirectional energy spectrum

$W_{k \perp} \sim \frac{B_{k}^{2}}{k_{\perp}} \propto k_{\perp}^{-3}$.

Again, one can observe steeper spectra $\propto\left(k_{\perp}^{-3} \div k_{\perp}^{-4}\right)$ if the strong KAW turbulence is not exactly axially symmetric around $B_{0}$.

Since $\gamma_{k \uparrow \uparrow}^{N L}$ depends on $k_{z}$ only through $B_{k}$, the $k_{z}$ dependence can appear via any functional form with the argument involving any combination of $k_{z}$ and $k_{\perp}$. Additional assumptions linking $k_{z}$ and $k_{\perp}$, such as the critical balance hypothesis, will be studied in another paper.

The strongly turbulent spectra of weakly dispersive counter-propagating KAWs can be found from Eq. (11) with $1 / \tau_{k}^{\mathrm{NL}} \sim \gamma_{k \uparrow \downarrow}^{\mathrm{NL}}$ given by Eq. (2):

$W_{k \perp} \sim \frac{B_{k}^{2}}{k_{\perp}} \propto k_{\perp}^{-7 / 3}$

\section{Strongly dispersive $k_{\perp} \rho_{\mathrm{p}}>1$ range of KAW turbulence}

In the strongly dispersive range of KAWs, where $k_{\perp} \rho_{\mathrm{p}}>1$, and which has been named the "KAW range" in the literature (see Schekochihin et al., 2009, and references therein), the rate of nonlinear interaction among co-propagating KAWs is (Voitenko, 1998a,b)

$\gamma_{k \uparrow \uparrow}^{\mathrm{NL}} \simeq 0.3 \Omega_{\mathrm{p}} \frac{V_{\mathrm{A}}}{V_{T p}}\left(k_{\perp} \rho_{\mathrm{p}}\right)^{2} \frac{B_{k}}{B_{0}}$.

For counter-propagating KAWs, the nonlinear interaction rate is almost the same,

$\gamma_{k \uparrow \downarrow}^{\mathrm{NL}} \simeq 0.2 \Omega_{\mathrm{p}} \frac{V_{\mathrm{A}}}{V_{T p}}\left(k_{\perp} \rho_{\mathrm{p}}\right)^{2} \frac{B_{k}}{B_{0}}$.

\subsection{Weak turbulence $\left(\gamma_{k}^{\mathrm{NL}} \ll \omega_{k}\right)$}

The weakly turbulent perpendicular wavenumber spectra of co-propagating KAWs behave as (Voitenko, 1998b):

$B_{k}^{2} \propto k_{\perp}^{-7 / 2} \quad$ (direct energy cascade),

$B_{k}^{2} \propto k_{\perp}^{-3} \quad$ (inverse enstrophy cascade).

Again, the nonlinear interaction amongcounter-propagating KAWs can be less efficient than that among co-propagating KAWs because of their shorter linear correlation times. Therefore, the omnidirectional spectra

$W_{k \perp} \propto k_{\perp}^{-5 / 2} \quad$ (direct energy cascade),

$W_{k \perp} \propto k_{\perp}^{-2} \quad$ (inverse enstrophy cascade)

can be produced by strongly dispersive KAWs in the weakly turbulent regime. Among these, the $\propto k_{\perp}^{-5 / 2}$ spectrum formed by the direct energy cascade is preferable, because the source is at largest scales. With local deviations from axial symmetry, one can expect steeper spectra $\propto k_{\perp}^{-2.5} \div k_{\perp}^{-3}$.

\subsection{Strong turbulence $\left(\omega_{k} \sim \gamma_{k}^{\mathrm{NL}}\right)$}

In the strong turbulence regime of co-propagating KAWs, the scaling of the magnetic field amplitude $B_{k}$ with $k_{\perp}$ is found from the condition Eq. (11) where $\tau_{k}^{\mathrm{NL}} \simeq 1 / \gamma_{k \uparrow \uparrow}^{\mathrm{NL}}$ with $\gamma_{k \uparrow \uparrow}^{\mathrm{NL}}$ given by (Eq. 14):

$B_{k} \propto k_{\perp}^{-2 / 3}$.

This results in the familiar omnidirectional power spectrum in $k_{\perp}$ :

$W_{k \perp} \sim \frac{B_{k}^{2}}{k_{\perp}} \propto k_{\perp}^{-7 / 3}$.

The "parallel" $k_{z} \| B_{0}$ spectrum

$W_{k \|} \propto k_{z}^{-2}$

follows from the critical balance condition. 


\section{MHD/kinetic Alfvén wave transition}

\subsection{Spectral kinks}

In the (Goldreich and Sridhar, 1995) MHD model, the nonlinear interaction rate at scale $\lambda_{\perp}$ in the plane $\perp B_{0}$ can be written as

$\gamma_{k}^{\mathrm{GS}} \simeq \frac{v_{\lambda \perp}}{\lambda_{\perp}} \simeq \frac{1}{2 \pi} k_{\perp} V_{\mathrm{A}} \frac{B_{k}}{B_{0}}$

where $v_{\lambda \perp}$ is the velocity and $B_{k}$ is the magnetic field amplitude at the scale $\lambda_{\perp}=2 \pi / k_{\perp}$. The corresponding MHD spectrum $B_{k}^{2} \propto k_{\perp}^{-2 / 3}$ follows from the independence of the energy flux through $k$. The omnidirectional spectrum $W_{k} \sim$ $B_{k}^{2} / k_{\perp} \propto k_{\perp}^{-5 / 3}$ is seen by satellites in the MHD range as the 1-D Doppler frequency spectrum.

As the MHD and WDR ranges have very different slopes, the first spectral kink should appear at the wavenumber where their respective nonlinear interaction rates are equal. Comparing the nonlinear rates, $\gamma_{k}^{\mathrm{GS}}=\gamma_{k \uparrow \downarrow}^{\mathrm{NL}}$, we obtain the spectral kink wavenumber $k_{\perp 1} \rho_{\mathrm{p}} \simeq 0.5$, at which the 1 -D spectrum should change from $-(3 / 2 \div 5 / 3)$ to $-(3 \div 4)$. The transition wavenumber for the $\gamma_{k}^{\mathrm{GS}}=\gamma_{k \uparrow \uparrow}^{\mathrm{NL}}$ transition is practically the same, $k_{\perp 1} \rho_{\mathrm{p}} \simeq 0.6$.

However, the above estimations did not take into account the weakening of the MHD nonlinear interactions by the dynamic alignment between velocity and magnetic perturbations (Boldyrev, 2005) and/or by the nonlocal decorrelation mechanism proposed by Gogoberidze (2007). In general, the interaction rate can be written as a reduced GS rate (RGS)

$\gamma_{k}^{\mathrm{RGS}} \simeq R_{\lambda \perp} \gamma_{k}^{\mathrm{GS}}$

with the scale-dependent reducing coefficient $R_{k \perp}$. Both Boldyrev's and Gogoberidze's phenomenologies give the same scaling for $R_{k \perp}$,

$R_{k \perp} \simeq \frac{v_{\lambda \perp}}{V_{\mathrm{N}}} \propto \lambda_{\perp}^{1 / 4}$,

but with different normalization velocities $V_{\mathrm{N}}$, such that the Boldyrev/Gogoberidze ratio $=v_{L} / V_{\mathrm{A}}$, where $v_{L}$ is the velocity amplitude at the driving scale $L$ (wavenumber $k_{L}$ ). Bearing in mind that the dynamic alignment saturates when approaching small scales, the actual value of the Gogoberidze coefficient can be larger even in the case $v_{L}<V_{\mathrm{A}}$. The reduced interaction rate proposed by Gogoberidze can be written as

$\gamma_{k}^{\mathrm{RGS}} \simeq R_{\lambda \perp}\left(\frac{v_{\lambda \perp}}{\lambda_{\perp}}\right) \simeq\left(\frac{k_{\perp}}{k_{L}}\right)^{-1 / 4}\left(\frac{1}{2 \pi} k_{\perp} V_{\mathrm{A}} \frac{B_{k}}{B_{0}}\right)$.

Given the typical width of the MHD inertial range in the solar wind $k_{b \perp} / k_{L} \sim 10^{3}$, we find that the interaction rate is reduced considerably in the vicinity of the spectral break, $\gamma_{k}^{\mathrm{RGS}} \simeq 0.25 \gamma_{k}^{\mathrm{GS}}$.

As the nonlocal decorrelation mechanism implies counterpropagating MHD waves, the counter-propagating KAWs should undergo the same decorrelation. But co-propagating KAWs do not suffer from such decorrelation, and therefore we consider here the MHD/kinetic transition dominated by the co-propagating KAWs. In addition, the co-propagating KAWs can keep in phase much longer than the counterpropagating KAWs. We therefore use Eq. (1) for the kinetic and Eq. (21) for the MHD interaction rate, and estimate the first spectral kink between shallow MHD spectra $-(3 / 2 \div 5 / 3)$ and steep weakly dispersive KAW spectra $-(3 \div 5)$ to be at

$k_{\perp 1} \rho_{\mathrm{p}} \simeq 0.6 \sqrt{R_{k \perp}}$.

With Gogoberidze's rate Eq. (22) $k_{\perp 1} \rho_{\mathrm{p}} \simeq 0.2$. But one should bear in mind that there are a number of factors, including a partial turbulence dissipation, which contribute to $R_{k \perp}$ and can make it smaller or larger than the Gogoberidze's value.

The second kink should appear between weakly $\left(k_{\perp}^{2} \rho_{\mathrm{p}}^{2} \ll 1\right)$ and strongly $\left(k_{\perp}^{2} \rho_{\mathrm{p}}^{2} \gg 1\right)$ dispersive regimes of the KAW turbulence at

$k_{\perp 2} \rho_{\mathrm{p}} \gtrsim 1$,

where we allow for a possible build-up of the steeper slope just above $k_{\perp} \rho_{\mathrm{p}}=1$ if the MHD/KAW transition is not yet completed at $k_{\perp}=\rho_{\mathrm{p}}^{-1}$. The spectral slope above $k_{\perp 2}$ is $-(2.5 \div 3)$, which is significantly shallower than in WDR.

\subsection{Spectral forms}

The steepness of the spectra in WDR depends on what kind of KAW turbulence picks up the turbulent cascade at $k_{\perp} \rho_{\mathrm{p}} \simeq$ 0.2 , weak or strong. If the critical balance condition holds at $k_{\perp} \rho_{\mathrm{p}} \simeq k_{\perp 1} \rho_{\mathrm{p}}$, then the turbulence of weakly dispersive KAWs is strong above $k_{\perp 1}$. In this case, strong KAW turbulence develops a steep energy spectrum $\propto k_{\perp}^{-3}$ in WDR, connecting shallower MHD $\left(\propto k_{\perp}^{-5 / 3}\right)$ and strongly dispersive KAW $\left(\propto k_{\perp}^{-7 / 3}\right)$ spectra. Significantly steeper spectra in both KAW ranges can be produced by the weak KAW turbulence and by local deviations from the azimuthal symmetry of the turbulence (up to about $\propto k_{\perp}^{-4.5}$ in WDR, and $\propto k_{\perp}^{-3}$ in the strongly dispersive range).

The transition to the weak turbulence regime may be facilitated by a partial wave dissipation via non-adiabatic ion acceleration/stochastic heating. Such partial dissipation is independent of $k_{z}$, but it does depend on $k_{\perp}$, and it reduces larger amplitudes at $k_{\perp}>k_{\perp \text { thr. }}$. In such a way, the critical balance between linear and nonlinear time scales is violated in favor of the weak turbulence regime. A weak turbulence cascade of KAWs develops above $k_{\perp \text { thr }}$ and establishes the steepest KAW spectra.

In both weak and strong turbulence regimes, the resulting spectra have two kinks, down and up, with the steepest slopes in between them in the weakly/mildly dispersive range. In 


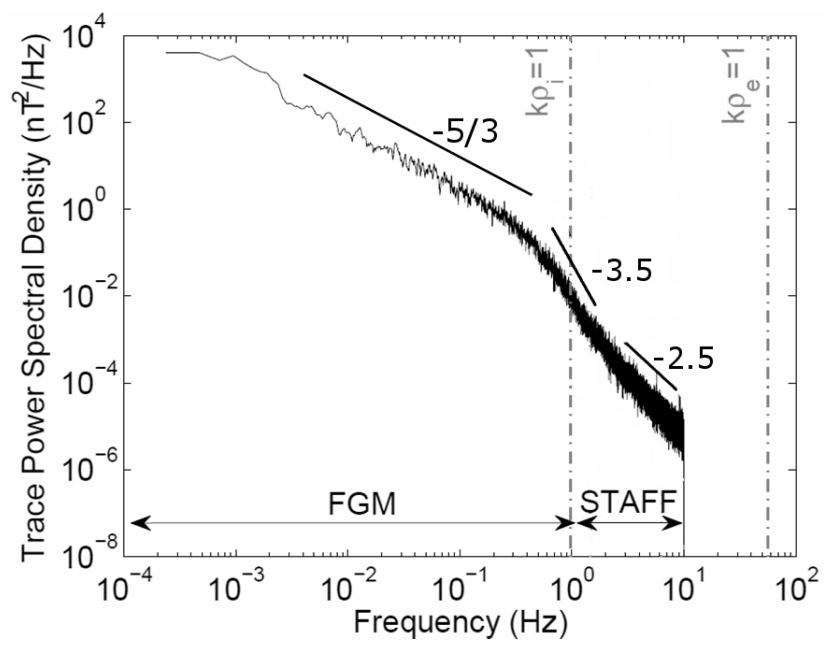

Fig. 1. Turbulent Alfvénic spectrum extending over three ranges, with the steepest slope in the weakly/mildly dispersive KAW range (interpretation of the spectrum shown in Fig. 1 by Chen et al., 2010).

general, the "non-dissipative" scenario is as follows: the turbulence, driven at a large MHD scale $L\left(k_{L}=2 \pi / L\right)$, develops the shallowest $\propto k_{\perp}^{-3 / 2} \div k_{\perp}^{-5 / 3}$ spectra in the MHD range $k_{L}<k_{\perp}<k_{\perp 1}$, then it proceeds as KAW turbulence with steep $\propto k_{\perp}^{-3} \div k_{\perp}^{-4.5}$ spectra in WDR $k_{\perp 1}<k_{\perp} \lesssim k_{\perp 2}$, and beyond $k_{\perp 2}$ it proceeds as KAW turbulence with $\propto k_{\perp}^{-7 / 3} \div k_{\perp}^{-3}$ spectrum in the strongly dispersive range $k_{\perp 2}<k_{\perp} \lesssim k_{\perp \text { de }}$. This last range may extend to the dissipative wavenumber $k_{\perp \text { de }}$ at the electron length scale (Sahraoui et al., 2010). The turbulence spectrum measured by Chen et al. (2010) can be explained by the combination of above spectra, as is shown in Fig. 1.

Consequently, the Alfvénic turbulence spectrum in the MHD/kinetic transition range has a universal double-kink form (2-k pattern). This 2-k spectral pattern is shown schematically in Fig. 2 for the case of a purely nonlinear non-dissipative transition. The (variable) slope of the weakly dispersive KAW spectrum depends on the ratio of turbulent energies cascading in strong and weak turbulent regimes, which can differ from case to case. The local slope should in principle lie between -3 and -4 . But the shallower $>-3$ spectra can be produced by a fraction of counter-propagating KAWs, and the steeper $<-4$ spectra can be produced by deviations from axial symmetry of the turbulence in the crossfield plane.

A similar 2-k pattern can in principle also occur for the dissipative transition, considered by many previous authors, but the conditions required for that are rather special. Namely, the relative dissipation rate (as compared to the nonlinear interaction rate) should be much stronger in the range $k_{\perp 1}<$ $k_{\perp}<k_{\perp 2}$ than in the range $k_{\perp}>k_{\perp 2}$. The presence of a high-wavenumber cascade and turbulence at $k_{\perp}>k_{\perp 2} \mathrm{im}$ plies a nonlinear transfer and a spectral flux across the range

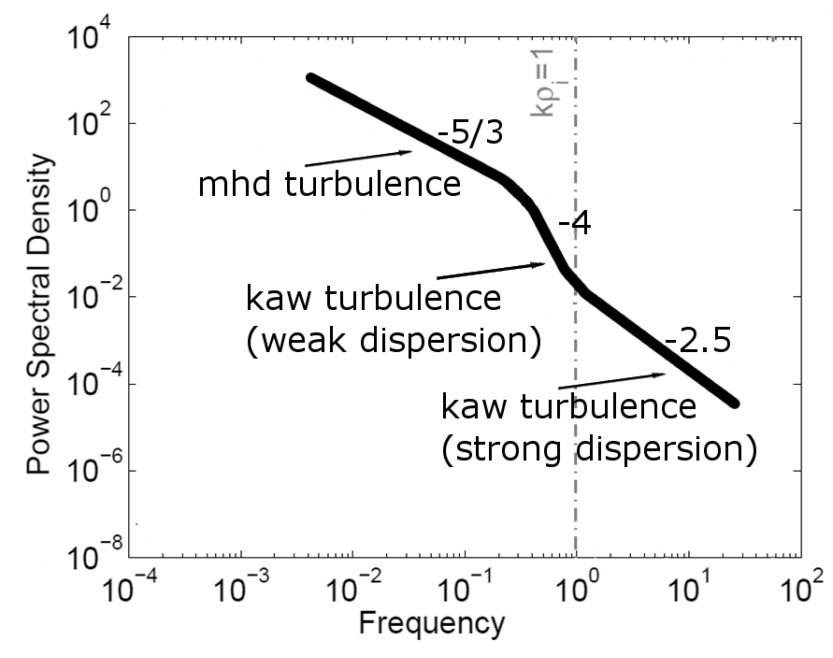

Fig. 2. Double-kink pattern produced by the MHD/WDR/strongly dispersive turbulence transitions. Kinetic Alfvén wave spectra are given for the case of a weakly turbulent regime. In the case of a strongly turbulent regime, spectral indices -4 and -2.5 should be replaced by -3 and $-7 / 3$, respectively.

$k_{\perp 1}<k_{\perp} \lesssim k_{\perp 2}$ as well, which means that nonlinear KAW interactions should be taken into account in any case.

The 2-k pattern described above can be noticed in many high-resolution high-frequency Cluster measurements (as reported by Kiyani et al., 2009; Sahraoui et al., 2010; Chen et al., 2010) and can also be noticed in some previous measurements where the frequency range extended to $1 \mathrm{~Hz}$ or a little higher (see e.g. ACE data reported by Smith et al., 2006, Fig. 1).

The relative importance of the effects due to dissipation versus weak turbulence versus strong turbulence in the MHD/kinetic transition can be different from case to case. The actual wavenumber range where non-adiabatic ion acceleration and related wave damping come into play is also variable. If the non-adiabatic ion acceleration and partial wave damping are active well below the apparent spectral kink, then the flatness should follow the trends as in Fig. 3 by Alexandrova et al. (2008a) for Cluster data. The threshold behavior suggests that it comes into play earlier for stronger spectral fluxes; it then weakens the MHD turbulence facilitating its transition to the weak KAW turbulence with its steeper spectra. This can explain a counter-intuitive observation by Smith et al. (2006) that the stronger fluxes are followed by the steeper spectra in the "dissipation range".

\section{Dissipation of KAWs}

In this section we discuss several pros and cons of basic dissipation mechanisms for KAWs, but their detailed investigation is postponed to the future. 
Wu and Yang (2007) considered self-consistent velocities of minor ion species in KAW solitons and found them distributed proportionally to the ion mass-to-charge ratio. However, these velocities cannot be interpreted as thermal ones (increasing temperature) because they contribute to the nonthermal line broadening rather than thermal line width. A non-adiabatic disconnection from the wave fields is needed for the ions to gain some additional energy after the wave has passed by. Such a process has been considered by Voitenko and Goossens (2004), who showed that ions can undergo strong non-adiabatic acceleration in the presence of KAWs. This acceleration requires a certain threshold-like amplitude/wavelength relation for KAWs (see a weakly dispersive example in Sect. 5.2), above which the ions get accelerated and the KAW dissipation is switched on. On the other hand, the acceleration is fast: it completes within a short time scale comparable to the ion gyroperiod and does not require a long stochastic walk for the ions to significantly gain energy (Voitenko and Goossens, 2004, 2006).

Chandran et al. (2010) have shown that another process related to non-adiabaticity (stochastic plasma heating) can absorb up to half of the turbulent cascade flux at $k_{\perp} \rho_{\mathrm{p}} \sim 1$. Chandran et al. assume the MHD nonlinear rate, which may be incorrect at $k_{\perp} \rho_{\mathrm{p}} \sim 1$ where the KAW nonlinear interaction is faster and can pass more energy to the high- $k_{\perp}$ range.

Yet another nonlinear interaction of the broadband Alfvénic turbulence with ions, via nonlinear Landau damping, was studied by Nariyuki et al. (2010), who showed that ion heating proceeds both along and across the background magnetic field and produces asymmetric ion velocity distributions. On the other hand, because of the quasi-linear plateau formation in velocity distribution functions, classic Landau damping can be highly reduced in the weakly collisional solar wind (Voitenko and Goossens, 2006; Rudakov et al., 2011).

\subsection{Landau damping}

The parallel components of the KAW electric $E_{z \mathrm{k}}$ and magnetic $B_{z \mathrm{k}}$ fields make the KAWs efficient in Cherenkov interaction with plasma species via the kinetic mechanisms of Landau and transit-time damping. Landau damping assuming Maxwellian distribution functions of plasma species that have been commonly used for estimating KAW dissipation. However, these mechanisms are based on resonant wave-particle interactions that depend strongly on the local parallel slopes of the particle velocity distributions $F_{\mathrm{S}}\left(V_{z}\right)$, at parallel velocities $V_{z}$ equal to the wave phase velocity $\omega_{k} / k_{z}$. In particular, quasi-linear diffusion smoothes resonant slopes and reduces Landau damping of KAWs by the factor $\left(1+\tau_{\mathrm{C}} / \tau_{\mathrm{KAW}}\right)^{-1}$ (Voitenko, 2006):

$\gamma_{\mathrm{L}}=\sum_{s} \gamma_{\mathrm{L}}^{\mathrm{M}}\left(1+\frac{\tau_{\mathrm{C}}}{\tau_{\mathrm{KAW}}}\right)^{-1}$ where $\gamma_{\mathrm{L}}^{\mathrm{M}}$ is the Maxwellian Landau damping rate, and $\tau_{\mathrm{KAW}}$ and $\tau_{\mathrm{C}}$ are the characteristic diffusion times of particles due to KAWs and Coulomb collisions, respectively. The summation in Eq. (25) is over plasma species $s$. KAWs tend to flatten $F_{\mathrm{S}}\left(V_{z}\right)$, while Coulomb collisions restore it back to Maxwellian; the balance between both results in Eq. (25).

The detailed analysis of Eq. (25) as a function of $k_{\perp}$ is quite complex (subject of a separate study). Our estimates, similar to those by Voitenko and Goossens (2006), show that for typical fluctuation levels $W_{\mathrm{f}} \sim 10^{-1} \mathrm{nT}^{2} / \mathrm{Hz}$ at $k_{\perp} \rho_{\mathrm{p}} \sim 1$ in the solar wind, $\tau_{\mathrm{C}} / \tau_{\mathrm{KAW}} \gg 1$ for both electrons and protons. Landau damping is thus highly reduced. The conclusion by Podesta (2009) that the KAW turbulence cannot reach electron scales in the solar wind, which was based on Maxwellian Landau damping, should therefore be reconsidered.

\subsection{Non-adiabatic threshold for turbulent dissipation}

The rate of the non-adiabatic cross-field acceleration of the ions " $i$ " by oblique Alfvén waves is (Voitenko and Goossens, 2004):

$\gamma_{\mathrm{n}-\mathrm{a}}^{2}=\Omega_{\mathrm{i}}^{2}\left[\frac{V_{\mathrm{A}}}{\Omega_{\mathrm{i}}}\left(\frac{c}{V_{\mathrm{A}}} \frac{E_{\perp}}{B_{\perp}}-\frac{V_{\mathrm{i} z}}{V_{\mathrm{A}}}\right) \frac{\partial}{\partial x} \frac{B_{\perp}}{B_{0}}-1\right]$,

where $V_{\mathrm{i} z}$ is the parallel ion velocity, $\Omega_{\mathrm{i}}$ is the ion-cyclotron frequency, and $E_{\perp}$ and $B_{\perp}$ are the Alfvénic electric and magnetic fluctuations, $E_{\perp} \perp B_{\perp}$.

Using $E_{\perp} / B_{\perp} \simeq V_{\mathrm{A}} / c$ in WDR, and ignoring a possible field-aligned streaming of ions, the threshold-like condition for this kind of wave-particle interaction, $\gamma_{n-a}^{2}>0$, can be written in the form

$\eta_{k}=k_{\perp} \delta_{\mathrm{p}} \frac{B_{k}}{B_{0}}>v_{i}$

where $v_{i}=\Omega_{\mathrm{i}} / \Omega_{\mathrm{p}}$ is the threshold value for the nonadiabatic factor $\eta_{k}$ above which the particular ion species $i$ is heated non-adiabatically. This condition applies to any particular ion species, but the related wave dissipation depends on all ion species and their parameters, like their abundances, temperatures, etc. Nevertheless, a condition for efficient wave dissipation can still be written in the form Eq. (27) with a non-adiabatic factor $\eta_{k}$ in the left hand side, but with a different threshold $v_{w}$ in the right hand side, which is not easy to find. One can guess that the wave threshold should be close to the acceleration threshold for the dominant ion species $v_{w} \sim v_{i}$. Anyway, even without knowing the exact threshold value $v_{w}$, it is possible to derive several useful scalings that can be tested observationally. So, for a power law scaling of magnetic amplitudes, $B_{k}^{2} \propto B_{k 1}^{2}\left(k_{\perp} / k_{\perp 1}\right)^{-q}$, we obtain the spectral dependence of $\eta_{k}$ :

$\eta_{k}=\eta_{k 1}\left(\frac{k_{\perp}}{k_{\perp 1}}\right)^{1-q / 2}>v_{w}$,

where $\eta_{k 1}$ is the non-adiabatic factor at the reference wavenumber $k_{\perp}=k_{\perp 1}$. For the sake of convenience we 


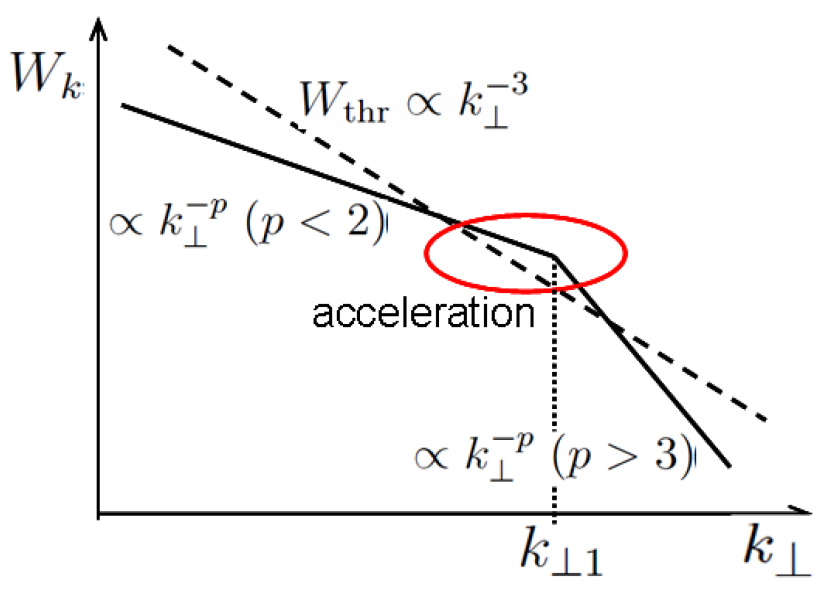

Fig. 3. Typical Alfvénic turbulent spectrum (solid line) in the weakly/mildly dispersive KAW range and a "threshold" turbulent spectrum (dashed line) required for non-adiabatic ion acceleration. Non-adiabatic acceleration is possible around the first spectral kink, where the turbulent spectral power rises above the threshold spectral power.

choose the reference wavenumber equal to the wavenumber of the first spectral kink $k_{\perp 1}$.

Since $q \simeq 2 / 3$ in the MHD range, $\eta_{k} \propto k_{\perp}^{2 / 3}$ grows with $k_{\perp}$ as long as $k_{\perp}<k_{\perp 1}$. But the situation is reversed in WDR, $k_{\perp}>k_{\perp 1}$, where $q \simeq 3$ and $\eta_{k}$ decreases with $k_{\perp}$ as $\eta_{k} \propto k_{\perp}^{-1 / 2}$. Such spectral $k_{\perp}$-dependence of $\eta_{k}$ indicates that the most favorable conditions for non-adiabatic ion acceleration and related wave dissipation are achieved in the vicinity of the first spectral kink, $k_{\perp} \simeq k_{\perp 1}$. This is shown schematically in Fig. 3, where we used the omnidirectional spectral representation $W_{k} \propto B_{k}^{2} / k_{\perp} \propto k_{\perp}^{-p}$ with $p=q+1$ for KAW spectra. The "threshold" spectrum

$W_{\mathrm{thr}} \propto \frac{B_{\mathrm{thr}}^{2}}{k_{\perp}} \propto k_{\perp}^{-3}$,

follows from the non-adiabatic condition Eq. (28), and has the same slope in both weakly and strongly dispersive ranges. But the measured spectrum has a variable slope: it is usually flatter $(p<2)$ in the MHD range, and steeper $(p>3)$ in WDR. The condition for the non-adiabatic ion acceleration by Alfvénic fluctuations is satisfied in the wavenumber range where the observed fluctuations' spectrum rises above the threshold one. As is seen from Fig. 3, with sufficient turbulence power, the non-adiabatic condition in spectral form, $W_{k}>W_{\mathrm{thr}}$, can be satisfied around the first spectral kink.

Once the threshold $\eta_{k}=v_{i}$ is exceeded in some wavenumber range for some ion species, these ions enter a regime of strong acceleration. In turn, because of its threshold-like character, the non-adiabatic ion heating provides a highly selective dissipation mechanism for waves, affecting only the strongest fluctuations with over-threshold amplitudes. In principle, the ability of turbulence to produce intermittent large-amplitude fluctuations increases the value of the driven parameter Eq. (28), where one should use the spectrum and amplitudes of the intermittent fluctuations instead of the regular turbulence spectrum. The eventual rate of the plasma heating and turbulence dissipation should follow from the balance between two processes: (i) production of the overthreshold intermittent fluctuations by the turbulence, and (ii) accommodation of turbulence energy by accelerated ions and its further redistribution into the bulk plasma. Helios observations have shown that the flatness (a measure of intermittency) increases with wavenumber (Alexandrova et al., 2008a), which progressively raises the non-adiabatic parameter above the value given by Eq. (28). Then, at some large enough wavenumber, the level of intermittent fluctuations can reach the non-adiabatic threshold, even if the regular turbulent level remains below it.

Dissipation due to non-adiabatic heating/acceleration tends to reduce the over-threshold fluctuations at every scale to the corresponding threshold value given by Eq. (27). Then, in accordance to Eq. (27), the upper bound for the reduced intermittent amplitudes scales as $B_{\mathrm{thr}}^{2} \propto k_{\perp}^{-2}$, and since the magnetic power spectrum in this range has shallower scaling $B_{k}^{2} \propto k_{\perp}^{-2 / 3}$, the flatness (and higher order normalized structure functions as well) should decrease with wavenumber in the MHD range below the first spectral kink. This can explain another interesting feature, the local decrease of the flatness in the spacecraft frequency range $0.02 \div 0.2 \mathrm{~Hz}$ (which is still below the apparent spectral kink) found by Alexandrova et al. (2008a) using Cluster data. We suggest that such behavior of the flatness may indicate a partial dissipation of Alfvén waves via non-adiabatic ion acceleration in the wavenumber range where $W_{k}>W_{\mathrm{thr}}$, illustrated in Fig. 3 .

In turn, highly anisotropic ion distributions are produced by non-adiabatic acceleration (Voitenko and Goossens, 2004), which can drive anisotropic ion-cyclotron instabilities redistributing energy further. Since non-adiabatic ion acceleration happens very fast, within a fraction of the corresponding ion gyroperiod, the quasi-stationary rate of turbulent dissipation will be determined by the ion-cyclotron instability increment. The situation is thus more complex here and opposite to that observed in hydrodynamics, where viscosity washes out the smallest amplitudes when approaching the dissipation range while large-amplitude fluctuations survive increasing intermittency. The behavior of the intermittency found by Alexandrova et al. (2008a) is not typical for linear Landau damping as well.

After the relative perpendicular/parallel power in the spectrum and the strength of the MHD interaction are reduced, the transition to weak KAW turbulence is made possible and leads to the steepest spectra in WDR. In the strongly dispersive range, Kiyani et al. (2009) found a monofractal (but still non-Gaussian) statistical behavior and suggested a "global scale-invariant dissipation". On the other hand, 
the turbulence is multifractal in the MHD range (see e.g. Marsch and Tu, 1997, about fractal scalings in the solar wind turbulence). The question is where and why the turbulence transforms from the multifractal state in the MHD range to the monofractal state in the kinetic range. To this end, it would be interesting to analyze intermittency and fractal properties in the weakly dispersive KAW range by means of Rank-Ordered Multifractal Analysis (ROMA) (see Chang et al., 2010, and references therein).

\section{Summary and discussion}

For the first time, a weakly dispersive range of the KAW turbulence is distinguished and studied in the context of the MHD/kinetic turbulence transition. We show that the KAW turbulence and its spectra in WDR differ significantly not only from conventional MHD Alfvénic turbulence, but also from strongly dispersive KAW turbulence. Namely, the nonlinear interaction of weakly dispersive KAWs is capable of producing steep spectra $\propto k_{\perp}^{-3} \div k_{\perp}^{-5}$ in the wavenumber range $k_{\perp 1}<k_{\perp}<\rho_{\mathrm{p}}^{-1}$, connecting shallow MHD spectra $\propto k_{\perp}^{-3 / 2} \div k_{\perp}^{-5 / 3}$ below the first spectral kink, $k_{\perp}<k_{\perp 1}$, and rather shallow $\propto k_{\perp}^{-7 / 3} \div k_{\perp}^{-3}$ spectra of strongly dispersive KAWs above the second spectral kink, $k_{\perp}>\rho_{\mathrm{p}}^{-1}$.

The universal spectral form resulting from such spectral dynamics in the transition range, the 2-k pattern, is shown schematically in Fig. 2. Turbulent spectra observed recently by the Cluster spacecraft often exhibit such a $2-\mathrm{k}$ pattern in the transition wavenumber range (see for example Fig. 1). It is still not certain what role Landau damping plays in producing such a steep spectral kink at $k_{\perp} \rho_{\mathrm{p}} \lesssim 1$. Any kind of kinetic dissipation in the weakly collisional solar wind should be self-consistently saturated at a reduced level by the local plateau formation in the velocity distribution functions of the plasma species (Voitenko and Goossens, 2006; Rudakov et al., 2011). At least a quasi-linear theory is needed to account for the particles' feedback on the energy input from the waves, and numerous previous estimations based on the Maxwellian Landau damping rate should be re-evaluated.

Podesta (2009) reported a significant flattening of the highfrequency parallel spectra and suggested this may be due to a plasma instability injecting a fraction of parallel propagating waves. On the other hand, this flattening can be produced by a transition to weak KAW turbulence, possessing (in an ideal case) a very shallow spectrum Eq. (10). However, because of many interfering factors, it is not certain if the parallel wavenumber spectrum Eq. (10) can be realized in the solar wind. The perpendicular wavenumber spectra are determined by the nonlinear interaction among perpendicular length scales and are thus quite robust. But the corresponding parallel wavenumber dynamics and spectra follow the perpendicular wavenumber dynamics and are often defined from a suitable functional form linking them to the perpendicular ones. This functional form may depend on a number of factors, including strength of the turbulence, partial turbulence dissipation, etc. In the extreme cases of weak and strong turbulence, the parallel dynamics is fixed, respectively, by the perpendicular one kinematically (via resonant conditions) and by adjusting linear and nonlinear time scales (via the critical balance condition).

One can expect a high variability of spectral slopes in WDR, resulting from a mixture of several "clean" spectra that can be produced by KAWs in this range. In addition, our analysis suggests that non-adiabatic and/or stochastic crossfield acceleration of solar wind ions are feasible mechanisms for a partial dissipation of KAWs operating in the vicinity of the first spectral kink. Both these mechanisms share the same non-adiabatic threshold and imply a selective dissipation of the over-threshold fluctuations with the largest amplitudes. This kind of dissipation reduces high-amplitude intermittent fluctuations and should therefore produce a local decrease of the flatness of the amplitude distribution of the fluctuations in the dissipation range. Although there are observational indications for such a behavior of the flatness (Alexandrova et al., 2008a, Fig. 3), this point needs further observational support.

It seems that the synergetic action of selective wave dissipation and weak turbulence of KAWs influences both the spectral kink positions and the spectral slopes, making them dependent of the turbulence level. Namely, $\eta_{k}$, product of the turbulent amplitude and corresponding wavenumber, is the parameter facilitating transition to weak KAW turbulence with its steeper spectra. As the spectral flux $\sim \eta_{k}^{3}$, the larger spectral fluxes imply larger $\eta_{k}$, which in turn imply steeper spectra in the weakly dissipative range. Such a counterintuitive trend was found by Smith et al. (2006).

On the other hand, in the vicinity of spectral kinks the nonadiabatic wave-particle interaction tends to reduce $\eta_{k}$ to a near-threshold value, which results in the scaling $B_{k 1} \sim k_{\perp 1}^{-1}$. This scaling offers an explanation for the observed spectral kink wavenumbers, which were found to be inversely proportional to the fluctuation amplitudes at the spectral kink positions (Markovskii et al., 2008).

Contrary to MHD Alfvén waves, the dispersion law of KAWs, even weakly dispersive, is not degenerate with respect to $k_{\perp}$. This makes 3-wave interactions possible with all 3 waves residing on the KAW branch, and there is no need for a zero- $k_{\|}, k_{\perp} \neq 0$ mode mediating the MHD turbulent cascade. Consequently, an additional spectrum of KAW turbulence can be created by the cascading enstrophy (dispersive part of energy). The energy and the enstrophy cascade in opposite directions from the injection wavenumber. As the turbulence of KAWs in the solar wind is driven at the largest MHD length scales, it naturally proceeds to smaller scales following a direct cascade route. In other environments, and with different positions of the driving scale, one may observe inverse (e.g. Lui et al., 2008) or dual spectral cascade. However, these are not easy to discriminate and describe in terms 
of cascades because of the non-local contaminations and scale mixing in a finite-size and highly variable environment, like in the Earth's plasma sheet (see Vörös et al., 2010).

Again, contrary to MHD Alfvén wave turbulence, KAW turbulence does not require pre-existing counter-propagating waves for efficient cascading. Nonlinear interaction among co-propagating KAWs is strong enough to establish a copropagating (completely imbalanced) KAW turbulence without involving the counter-propagating KAWs. If the copropagating KAW turbulence develops in some wavenumber range (e.g. at $k_{\perp 1}<k_{\perp}<\rho_{\mathrm{p}}^{-1}$ ), then the ratio of sunward/anti-sunward Poynting fluxes should be frozen and remain approximately constant at these wavenumbers. This would provide another observational benchmark for KAW turbulence, but we are not aware of such observations at $k_{\perp} \simeq \rho_{\mathrm{p}}^{-1}$ so far.

Acknowledgements. This work was supported in part by the STCE (Solar-Terrestrial Center of Excellence) under the project "Fundamental science". Some results of this paper were presented and discussed at the Turbulence and Multifractals Workshop (8-11 June 2010, Brussels, Belgium).

Edited by: H. Lamy

Reviewed by: G. Gogoberidze and another anonymous referee

\section{References}

Alexandrova, O., Carbone, V., Veltri, P., and L. Sorriso-Valvo: Small-scale energy cascade of the solar wind turbulence, Astrophys. J., 674, 1153-1157, doi:10.1086/524056, 2008a.

Alexandrova, O., Alexandrova, O., Lacombe, C., and Mangeney, A.: Spectra and anisotropy of magnetic fluctuations in the Earth's magnetosheath: Cluster observations, Ann. Geophys., 26, 35853596, doi:10.5194/angeo-26-3585-2008, 2008b.

Alexandrova, O., Saur, J., Lacombe, C., Mangeney, A., Mitchell, J., Schwartz, S. J., and Robert, P.: Universality of Solar-Wind Turbulent Spectrum from MHD to Electron Scales, Phys. Rev. Lett., 103, 165003, doi:10.1103/PhysRevLett.103.165003, 2009.

Alexandrova, O., Saur, J., Lacombe, C., Mangeney, A., Schwartz, S. J., Mitchell, J., Grappin, R., and Robert, P.: Solar wind turbulent spectrum from MHD to electron scales, AIP Conference Proceedings, 1216, 144-147, 2010.

Bale, S. D., Kellogg, P. J., Mozer, F. S., Horbury, T. S. and Reme, H.: Measurement of the electric fluctuation spectrum of magnetohydrodynamic turbulence, Phys. Rev. Lett., 94(21), 215002, doi:10.1103/PhysRevLett.94.215002, 2005.

Boldyrev, S.: On the Spectrum of Magnetohydrodynamic Turbulence, Astrophys. J., 626, L37-L40, 2005.

Carbone, V., Perri, S., Yordanova, E., Veltri, P., Bruno, R., Khotyaintsev, Y., and André, M.: Sign-Singularity of the Reduced Magnetic Helicity in the Solar Wind Plasma, Phys. Rev. Lett., 104, 181101, doi:10.1103/PhysRevLett.104.181101, 2010.

Chandran, B. D. G., Li, B., Rogers, B. N., Quataert, E., and Germaschewski, K.: Perpendicular Ion Heating by Low-frequency Alfvén-wave Turbulence in the Solar Wind, Astrophys. J., 720, 503-515, 2010.
Chang, T., Wu, C. C., Podesta, J., Echim, M., Lamy, H., and Tam, S. W. Y.: ROMA (Rank-Ordered Multifractal Analyses) of intermittency in space plasmas a brief tutorial review, Nonlin. Processes Geophys., 17, 545-551, doi:10.5194/npg-17-545-2010, 2010.

Chen, C. H. K., Horbury, T. S., Schekochihin, A. A., Wicks, R. T., Alexandrova, O., and Mitchell, J.: Anisotropy of Solar Wind Turbulence between Ion and Electron Scales, Phys. Rev. Lett., 104, 255002, doi:10.1103/PhysRevLett.104.255002, 2010.

Gogoberidze, G.: On the nature of incompressible magnetohydrodynamic turbulence, Phys. Plasmas, 14, 022304-022304-11, doi:10.1063/1.2437753, 2007.

Goldreich, P. and Sridhar, S.: Toward a theory of interstellar turbulence. 2: Strong alfvenic turbulence, Astrophys. J., 438, 763$775,1995$.

Hasegawa, A. and Chen, L.: Kinetic processes in plasma heating by resonant mode conversion of Alfvé in wave, Phys. Fluids, 19 (12), 1924-1934, 1976.

Howes, G. G., Cowley, S. C., Dorland, W., Hammett, G. W., Quataert, E., and Schekochihin, A. A.: A model of turbulence in magnetized plasmas: Implications for the dissipation range in the solar wind, J. Geophys. Res., 113, A05103, doi:10.1029/2007JA012665, 2008.

Kiyani, K. H., Chapman, S. C., Khotyaintsev, Yu. V., Dunlop, M. W., and Sahraoui, F.: Global Scale-Invariant Dissipation in Collisionless Plasma Turbulence, Phys. Rev. Lett., 103, 075006, doi:10.1103/PhysRevLett.103.075006, 2009.

Leamon, R. J., Smith, C. W., Ness, N. F., and Wong, H. K.: Dissipation range dynamics: Kinetic Alfvén waves and the importance of electron $\beta_{e}$, J. Geophys. Res., 104, 22331-22344, doi:10.1029/1999JA900158, 1999.

Lui, A. T. Y., Yoon, P. H., Mok, and C., Ryu, C.-M.: Inverse cascade feature in current disruption, J. Geophys. Res., 113, A00C06, doi:10.1029/2008JA013521, 2008.

Luo, Q. Y. and Wu, D. J.: Observations of Anisotropic Scaling of Solar Wind Turbulence, Astrophys. J., 714, L138-L141, 2010.

Mangeney, A., Lacombe, C., Maksimovic, M., Samsonov, A. A., Cornilleau-Wehrlin, N., Harvey, C. C., Bosqued, J.-M., and Trávnc(ek, P.: Cluster observations in the magnetosheath Part 1: Anisotropies of the wave vector distribution of the turbulence at electron scales, Ann. Geophys., 24, 3507-3521, doi:10.5194/angeo-24-3507-2006, 2006.

Markovskii, S. A., Vasquez, B. J. and Smith, C. W.: Statistical analysis of the high-frequency spectral break of the solar wind turbulence at 1AU, Astrophys. J., 675, 1576-1583, 2008.

Marsch, E. and Tu, C.-Y.: Intermittency, non-Gaussian statistics and fractal scaling of MHD fluctuations in the solar wind, Nonlin. Processes Geophys., 4, 101-124, doi:10.5194/npg-4-101-1997, 1997.

Matthaeus, W. H., Goldstein, M. L., and Roberts, D. A.: Evidence for the presence of quasi-two-dimensional nearly incompressible fluctuations in the solar wind, J. Geophys. Res. (ISSN 01480227), 95, Dec. 1, 20673-20683, 1990.

Narita, Y., Gary, S. P., Saito, S., Glassmeier, K.-H., and Motschmann, U.: Dispersion relation analysis of solar wind turbulence, Geophys. Res. Lett., 38, L05101, doi:10.1029/2010GL046588, 2011.

Nariyuki, Y., Hada, T., and Tsubouchi, K.: Heating and acceleration of ions in nonresonant Alfvénic turbulence, Phys. Plasmas, 17, 
072301-072301-5, doi:10.1063/1.3449592, 2010.

Osman, K. T. and Horbury, T. S.: Multi-spacecraft measurement of anisotropic power levels and scaling in solar wind turbulence, Ann. Geophys., 27, 3019-3025, doi:10.5194/angeo-273019-2009, 2009.

Perri, S., Carbone, V., and Veltri, P.: Where Does Fluid-like Turbulence Break Down in the Solar Wind?, Astrophys. J., 725, L52L55, 2010.

Podesta, J. J.: Dependence of Solar-Wind Power Spectra on the Direction of the Local Mean Magnetic Field, Astrophys. J., 698, 986-999, 2009.

Podesta, J. J., Borovsky, J. E., and Gary, S. P.: A Kinetic Alfvén Wave Cascade Subject to Collisionless Damping Cannot Reach Electron Scales in the Solar Wind at 1 AU, Astrophys. J., 712, 685-691, 2010.

Rudakov, L., Mithaiwala, M., Ganguli, G., and Crabtree, C.: Linear and nonlinear Landau resonance of kinetic Alfvén waves: Consequences for electron distribution and wave spectrum in the solar wind, Phys. Plasmas, 18, 012307-012307-10, doi:10.1063/1.3532819, 2011.

Sahraoui, F., Goldstein, M. L., Robert, P., and Khotyaintsev, Yu. V.: Evidence of a Cascade and Dissipation of Solar-Wind Turbulence at the Electron Gyroscale, Phys. Rev. Lett., 102, 231102, doi:10.1103/PhysRevLett.102.231102, 2009.

Sahraoui, F., Goldstein, M. L., Belmont, G., Canu, P., and Rezeau, L.: Three Dimensional Anisotropic k Spectra of Turbulence at Subproton Scales in the Solar Wind, Phys. Rev. Lett., 105, 131101, doi:10.1103/PhysRevLett.105.131101, 2010.

Saito, S., Gary, S. P., and Narita, Y.: Wavenumber spectrum of whistler turbulence: Particle-in-cell simulation, Phys. Plasmas, 17, 122316-122316-7, doi:10.1063/1.3526602, 2010.

Salem, C., Mangeney, A., Bale, S. D., and Veltri, P.: Solar Wind Magnetohydrodynamics Turbulence: Anomalous Scaling and Role of Intermittency, Astrophys. J., 702, 537-553, 2009.
Schekochihin, A. A., Cowley, S. C., Dorland, W., Hammett, G. W., Howes, G. G., Quataert, E., and Tatsuno, T.: Astrophysical gyrokinetics: Kinetic and fluid turbulent cascades in magnetized weakly collisional plasmas, Astrophys. J. Suppl., 182, 310-377, doi:10.1088/0067-0049/182/1/310, 2009.

Smith, C. W., Hamilton, K., Vasquez, B. J., and Leamon, R. J.: Dependence of the Dissipation Range Spectrum of Interplanetary Magnetic Fluctuationson the Rate of Energy Cascade, Astrophys. J. Lett., 645, L85-L88, doi:10.1086/506151, 2006.

Sorriso-Valvo, L., Carbone, V., Veltri, P., Consolini, G., and Bruno, R.: Intermittency in the solar wind turbulence through probability distribution functions of fluctuations, Geophys. Res. Lett., 26, 1801-1804, 1999.

Voitenko, Yu. M.: Three-wave coupling and parametric decay of kinetic Alfvén waves, J. Plasma Phys. 60, 497-514, doi:10.1017/S0022377898007090, 1998a.

Voitenko, Yu. M.: Three-wave coupling and weak turbulence of kinetic Alfvén waves, J. Plasma Phys. 60, 515-527, doi:10.1017/S0022377898007107, 1998b.

Voitenko, Y. and Goossens, M.: Cross-Field Heating of Coronal Ions by Low-Frequency Kinetic Alfvén Waves, Astrophys. J. 605, L149-L152, doi:10.1086/420927, 2004.

Voitenko, Y. and Goossens, M.: Energization of Plasma Species by Intermittent Kinetic Alfvén Waves, Space Sci. Rev., 122, 255270, 2006.

Vörös, Z., Runov, A., Leubner, M. P., Baumjohann, W., and Volwerk, M.: Is current disruption associated with an inverse cascade?, Nonlin. Processes Geophys., 17, 287-292, doi:10.5194/npg-17-287-2010, 2010.

Wu, D. J. and Yang, L.: Nonlinear Interaction of Minor Heavy Ions with Kinetic Alfvén Waves and Their Anisotropic Energization in Coronal Holes, Astrophys. J., 659, 1693-1701, 2007. 